\title{
Symplectic embeddings of ellipsoids in dimension greater than four
}

\author{
Olguta Buse \\ RICHARD HIND
}

\begin{abstract}
We study symplectic embeddings of ellipsoids into balls. In the main construction, we show that a given embedding of $2 m$-dimensional ellipsoids can be suspended to embeddings of ellipsoids in any higher dimension. In dimension 6 , if the ratio of the areas of any two axes is sufficiently large then the ellipsoid is flexible in the sense that it fully fills a ball. We also show that the same property holds in all dimensions for sufficiently thin ellipsoids $E(1, \ldots, a)$. A consequence of our study is that in arbitrary dimension a ball can be fully filled by any sufficiently large number of identical smaller balls, thus generalizing a result of Biran valid in dimension 4 .
\end{abstract}

\section{Introduction}

Let $E\left(a_{1}, \ldots, a_{n}\right) \subset \mathbb{R}^{2 n}$ be the ellipsoid

$$
E\left(a_{1}, \ldots, a_{n}\right)=\left\{\sum_{i=1}^{n} \frac{\pi\left(x_{i}^{2}+y_{i}^{2}\right)}{a_{i}} \leq 1\right\} .
$$

Ellipsoids inherit a symplectic structure from the standard form $\omega_{0}=\sum_{i=1}^{n} d x_{i} \wedge d y_{i}$ on $\mathbb{R}^{2 n}$. Then, in our notation, the ball of capacity $c$ is written

$$
B^{2 n}(c)=E(c, \ldots, c) .
$$

Let us also write $\lambda E\left(a_{1}, \ldots, a_{n}\right)$ and $\lambda B(c)$ for the ellipsoid $E\left(\lambda a_{1}, \ldots, \lambda a_{n}\right)$ and ball $B(\lambda c)$ respectively. Throughout the paper the notation

$$
E\left(a_{1}, \ldots, a_{n}\right) \hookrightarrow E\left(b_{1}, \ldots, b_{n}\right)
$$

will mean that for all $\lambda>1$ there exists a symplectic embedding $E\left(a_{1}, \ldots, a_{n}\right) \hookrightarrow$ $\lambda E\left(b_{1}, \ldots, b_{n}\right)$.

We are interested in the problem of determining when there exists a symplectic embedding from a given ellipsoid into (an arbitrarily small neighborhood of) the ball of capacity $c$. 
This problem has been completely solved when $n=2$, that is, in dimension 4 , in the sense that the function

$$
g(a):=\inf \left\{c \mid E(1, a) \hookrightarrow B^{4}(c)\right\}
$$

is described by McDuff and Schlenk [21, Theorem 1.1.2]; see our Proposition 2.6.

Here we begin a systematic study of the corresponding functions in higher dimensions. The main construction that we introduce allows us to extend known results on embeddings in low dimension to higher dimensional embeddings:

\section{Proposition 1.1 Suppose that}

Then also

$$
E\left(a_{1}, \ldots, a_{m}\right) \hookrightarrow E\left(a_{1}^{\prime}, \ldots, a_{m}^{\prime}\right) .
$$

$$
E\left(a_{1}, \ldots, a_{m}, a_{m+1}, \ldots, a_{n}\right) \hookrightarrow E\left(a_{1}^{\prime}, \ldots, a_{m}^{\prime}, a_{m+1}, \ldots, a_{n}\right)
$$

for any values $a_{m+1}, \ldots, a_{n}$.

We will focus especially on dimension 6 , where the problem is to describe the function of two variables

$$
f(a, b):=\inf \left\{c \mid E(1, a, b) \hookrightarrow B^{6}(c)\right\} .
$$

Note that by symmetry and rescaling we may assume that $1 \leq a \leq b$.

We are able to describe $f$ completely in particular when $a^{2}+b^{2} \leq 4$ and also when $a^{2}+b^{2} \geq 1.41 \times 10^{101}$. In other words we have optimal embedding results when the ellipsoid is either relatively close to a ball or in the other extreme when it is, up to scale, contained in a relatively small neighborhood of a 4-dimensional ellipsoid. The results for $a, b$ small are contained in the sequence of Lemmas 2.7, 2.8, 2.9, 2.11. More known values of $f(a, b)$ are illustrated in Figure 1. The result for $a$ or $b$ large is perhaps the main result of our paper and can be stated as follows.

Theorem 1.2 If $a^{2}+b^{2} \geq 1.41 \times 10^{101}$ then $E(1, a, b) \hookrightarrow B\left((a b)^{1 / 3}\right)$.

This means that in the given range we have volume filling embeddings, that is, the only obstruction to embedding this class of ellipsoids into a ball comes from their volumes. In dimension 4 the analogous result is that $E(1, a) \hookrightarrow B(\sqrt{a})$ provided that $a>8 \frac{1}{36}$.

A consequence of Theorem 1.2 is a full packing (or filling) result for higher dimensional balls. Let $\bigsqcup_{k} B(c)$ be the disjoint union of $k$ standard $2 n$-dimensional balls of radius $r$ and capacity $c=\pi r^{2}$. 


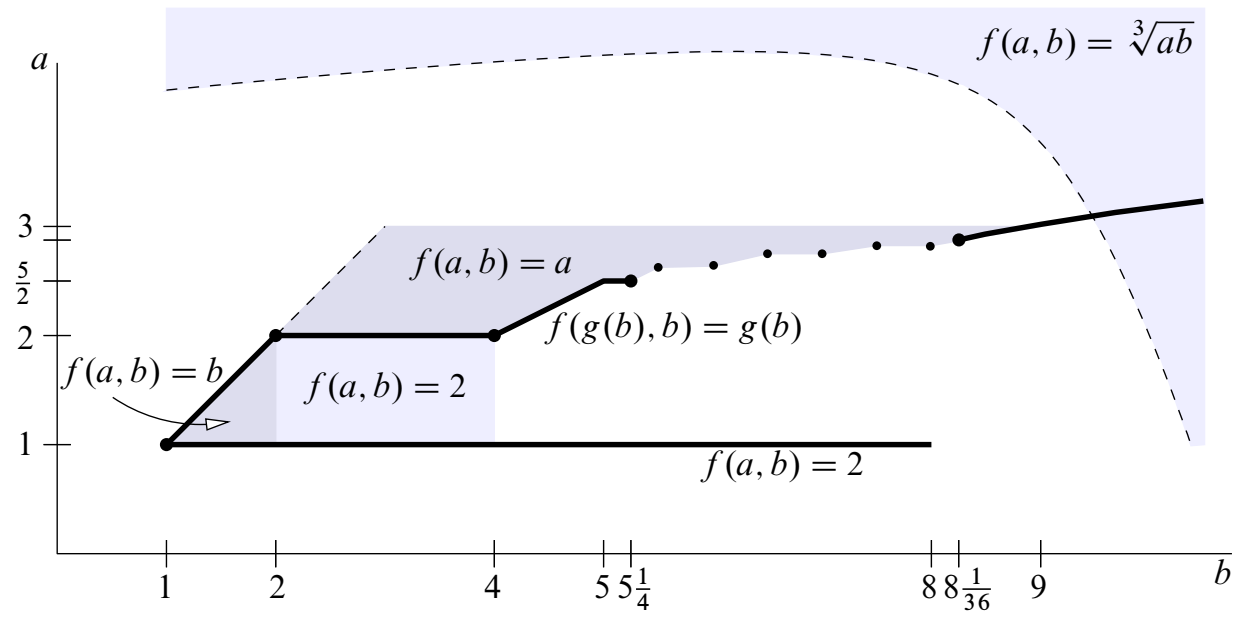

Figure 1: Known values of $f(a, b)$

Theorem 1.3 For any natural number $n \geq 3$ there exists a number $M_{n}$ such that for all $k \geq M_{n}$,

$$
\bigsqcup_{k} B\left(1 / k^{1 / n}\right) \hookrightarrow B^{2 n}(1) .
$$

In other words, the round ball can be fully filled by a disjoint union of any number $k \geq M_{n}$ of identical balls. For the definition of $M_{n}$, see Definition 3.6.

The $k$-th packing number of a compact, $2 n$-dimensional, symplectic manifold $(M, \omega)$ is

$$
p_{k}(M, \omega)=\frac{\sup _{c} \operatorname{Vol}\left(\bigsqcup_{k} B(c)\right)}{\operatorname{Vol}(M, \omega)},
$$

where the supremum is taken over all $c$ for which there exist a symplectic embedding of $\bigsqcup_{k} B(c)$ into $(M, \omega)$. Naturally, $p_{k}(M, \omega) \leq 1$. When $p_{k}(M, \omega)=1$ we say that $(M, \omega)$ admits a full packing by $k$ balls, otherwise we say that there is a packing obstruction.

Although no general tools are known to compute those invariants for arbitrary symplectic manifolds, some results can be derived from complex algebraic geometry using the theory of $J$-holomorphic curves. A first result that follows from Gromov [10] is that $p_{i}\left(\mathbb{C P}^{n}\right)<1$ for any $1<i<2^{n}$. McDuff and Polterovich [20] computed $p_{i}\left(\mathbb{C P}^{2}\right)$ for $i \leq 9$. They also proved that $p_{i}\left(\mathbb{C P}^{n}\right)=1$ whenever $i=k^{n}$ and that $\lim _{i \rightarrow \infty} p_{i}(M, \omega)=1$ for any compact symplectic manifold. Such results led to the natural question of whether the sequence $p_{i}(M, \omega)$ is eventually stable, that is, whether there is a number $N_{\text {stab }}(M, \omega)$ such that $p_{i}(M, \omega)=1$ for all $i \geq N_{\text {stab }}(M, \omega)$. To date, this remains an interesting open question (see Biran [3] and Cieliebak et al [5] 
for a complete discussion). When $M$ is four dimensional, results of McDuff [17] and Lalonde and McDuff [12] regarding the structure of symplectic ruled surfaces and introducing inflation techniques then opened the way to a thorough study of the packing numbers. This study was done by $\mathrm{P}$ Biran in a sequence of papers $[1 ; 2]$ which answered the stability question positively in the cases of closed symplectic 4manifolds whose symplectic forms (after rescaling) are in rational cohomology classes. His techniques allowed him to obtain upper and lower bounds for $N_{\text {stab }}\left(M^{4}, \omega\right)$ which can be explicitly computed in some cases. In particular, he showed that $N_{\text {stab }}\left(\mathbb{C P}^{2}\right) \leq 9$ which, in view of McDuff and Polterovich's results, is sharp. Although suspected to be true, until now there were no results in the literature proving packing stability for a symplectic manifold of dimension larger than 4 . Theorem 1.3 above shows that balls admit full packings by a sufficiently large number of disjoint identical balls. As the affine part of $\mathbb{C P}^{n}$ is a ball, in this language Theorem 1.3 gives the following.

Theorem 1.4 Consider $\left(\mathbb{C} \mathbb{P}^{n}, \omega\right)$ with the symplectic form induced by the FubiniStudy metric. Then $\mathbb{C P}^{n}$ has packing stability; indeed

$$
p_{i}\left(\mathbb{C P}^{n}\right)=1 \quad \text { when } i \geq M_{n}
$$

Probably the bound $M_{n}$ is not optimal, we briefly discuss this in Remark 4.4.

Outline of the paper In Section 2 we describe our basic embedding construction in Proposition 1.1 and apply it to deduce various values of $f(a, b)$, in particular for $a, b$ sufficiently small. We remark that E Opshtein [22] has also given a construction for embedding ellipsoids. The focus of his work is perhaps embeddings into closed manifolds, but there is still some overlap with our own results. We mention this also in Section 2.

Section 3 gives the proof of Theorem 1.2 and also constructs some volume filling embeddings of balls by ellipsoids in any dimension.

Theorem 1.3 is proven in Section 4, by combining the volume filling results of Section 3 with a construction from toric geometry.

Acknowledgements The second author would like to thank Kaoru Ono for an enlightening discussion on toric decompositions, and both authors thank Dusa McDuff and an anonymous referee for helpful comments on the text. 


\section{Embedding ellipsoids}

\subsection{The construction}

Here we give the proof of our basic embedding construction. Before giving a formal proof of Proposition 1.1 we outline the general idea. Identifying each $\mathbb{R}^{2 n}$ with $\mathbb{C}^{n}$, let $H_{s}: \mathbb{C}^{m} \times[0,1] \rightarrow \mathbb{R}$ be a 1 -parameter family of possibly time-dependent Hamiltonian functions on $\mathbb{C}^{m}$ and set $H(z, s, t)=H_{s}(z, t)$, so $H: \mathbb{C}^{m} \times \mathbb{R} \times[0,1] \rightarrow \mathbb{R}$. Then for any function $f: \mathbb{C}^{n-m} \rightarrow \mathbb{R}$, the function $H(z, f, t)$ can be thought of as a Hamiltonian $G$ on $\mathbb{C}^{n}$. Let $\pi: \mathbb{C}^{n} \rightarrow \mathbb{C}^{n-m}$ be the projection on the last $n-m$ complex coordinates, then $F=f \circ \pi: \mathbb{C}^{n} \rightarrow \mathbb{R}$ is an integral of the motion of $G$. Indeed, at all times $t$, we have $\{G, F\}=d G\left(X_{F}\right)=\frac{\partial H}{\partial s} d f\left(X_{f}\right)=0$. Therefore the flow of $G$ preserves the level sets of $F$, and restricted to a level $\Sigma_{c}=\{F=c\}=$ $\mathbb{C}^{m} \times\{f=c\}$ the first $m$ (complex) components of the flow are exactly those of the Hamiltonian $H_{c}$. In other words, let $\Phi$ be the time 1 flow of $G$ and $\phi_{c}$ be the time 1 flow of $H_{c}$. Then if $(z, w) \in \mathbb{C}^{m} \times \mathbb{C}^{n-m}=\mathbb{C}^{n}$ has $F(z, w)=f(w)=c$, we have $\Phi(z, w)=\left(\phi_{c}(z), w^{\prime}\right)$ where $f\left(w^{\prime}\right)=c$. Thus a domain $D \subset \mathbb{C}^{n}$ whose fibers $\pi^{-1}(w) \cap D=D_{w}=D_{f(w)}$ depend only on $f(w)$ will be mapped under $\Phi$ to a domain $D^{\prime}$ with fibers $\pi^{-1}(w) \cap D^{\prime}=\phi_{f(w)}\left(D_{f(w)}\right)$.

Returning to Proposition 1.1 , and still identifying $\mathbb{R}^{2 n}$ with $\mathbb{C}^{n}$, we can write

$$
E\left(a_{1}, \ldots, a_{n}\right)=\left\{\left(z_{1}, \ldots, z_{n}\right) \in \mathbb{C}^{n} \mid \frac{\pi\left|z_{1}\right|^{2}}{a_{1}}+\cdots+\frac{\pi\left|z_{n}\right|^{2}}{a_{n}} \leq 1\right\} .
$$

Using the notation above, the fibers of $E\left(a_{1}, \ldots, a_{n}\right)$ over $\mathbb{C}^{n-m}$ are ellipsoids $r E\left(a_{1}, \ldots, a_{m}\right)$ where $r=1-\pi\left|z_{m+1}\right|^{2} / a_{m+1}-\cdots-\pi\left|z_{n}\right|^{2} / a_{n}$. Roughly speaking, we will apply our general idea to a 1-parameter family of Hamiltonian functions $H_{r}$ whose corresponding flows map $r E\left(a_{1}, \ldots, a_{m}\right)$ into $r E\left(a_{1}^{\prime}, \ldots, a_{m}^{\prime}\right)$.

Proof of Proposition 1.1 Fixing a $\lambda>1$, it is required to show that there exists a symplectic embedding $E\left(a_{1}, \ldots, a_{m}, a_{m+1}, \ldots, a_{n}\right) \rightarrow \lambda E\left(a_{1}^{\prime}, \ldots, a_{m}^{\prime}, a_{m+1}, \ldots, a_{n}\right)$.

By hypothesis, we have $E\left(a_{1}, \ldots, a_{m}\right) \hookrightarrow E\left(a_{1}^{\prime}, \ldots, a_{m}^{\prime}\right)$. By the Extension after Restriction Principle (see Schlenk [23, page 7]) our hypothesis implies that there exists a $\mu>1$ and a Hamiltonian diffeomorphism mapping $\mu E\left(a_{1}, \ldots, a_{m}\right) \rightarrow$ $\lambda E\left(a_{1}^{\prime}, \ldots, a_{m}^{\prime}\right)$. Suppose that this Hamiltonian diffeomorphism is the time 1 flow corresponding to a Hamiltonian function $H: \mathbb{C}^{m} \times[0,1] \rightarrow \mathbb{R}$.

Observe that for any $r>0$ the Hamiltonian $H_{r}$ defined by $H_{r}(z, t)=r H(z / \sqrt{r}, t)$ for $z \in \mathbb{C}^{m}$ generates a flow with time 1 map taking $r \mu E\left(a_{1}, \ldots, a_{m}\right) \rightarrow r \lambda E\left(a_{1}^{\prime}, \ldots, a_{m}^{\prime}\right)$. 
(For this, recall that in our notation the map $\left(z_{1}, \ldots, z_{m}\right) \mapsto\left(\sqrt{r} z_{1}, \ldots, \sqrt{r} z_{m}\right)$ takes $E\left(a_{1}, \ldots, a_{m}\right)$ onto $r E\left(a_{1}, \ldots, a_{m}\right)$.)

For $z \in \mathbb{C}^{n}$, let

$$
r(z)=r\left(z_{m+1}, \ldots, z_{n}\right)=1-\frac{\pi\left|z_{m+1}\right|^{2}}{\mu a_{m+1}}-\cdots-\frac{\pi\left|z_{n}\right|^{2}}{\mu a_{n}} .
$$

Define

$$
K\left(z_{1}, \ldots, z_{n}, t\right)=H_{r\left(z_{m+1}, \ldots, z_{n}\right)}\left(z_{1}, \ldots, z_{m}, t\right)
$$

for $z$ with $\pi\left|z_{m+1}\right|^{2} / a_{m+1}+\cdots+\pi\left|z_{n}\right|^{2} / a_{n} \leq 1$ and extend the function arbitrarily to the remainder of $\mathbb{C}^{n} \times[0,1]$. Note that $\pi\left|z_{m+1}\right|^{2} / a_{m+1}+\cdots+\pi\left|z_{n}\right|^{2} / a_{n} \leq 1$ implies that $r(z) \geq 1-1 / \mu>0$ and so $K$ is well defined. We claim that $K$ generates a Hamiltonian diffeomorphism $\phi$ as required.

More precisely, we make the following claim. Suppose that $z \in \mathbb{C}^{n}$ is such that $\pi\left|z_{m+1}\right|^{2} / a_{m+1}+\cdots+\pi\left|z_{n}\right|^{2} / a_{n}=k \leq 1$ and $\phi(z)=w=\left(w_{1}, \ldots, w_{n}\right)$. Then

(1) $\pi\left|w_{m+1}\right|^{2} / a_{m+1}+\cdots+\pi\left|w_{n}\right|^{2} / a_{n}=k$;

(2) $\pi\left|w_{1}\right|^{2} / a_{1}^{\prime}+\cdots+\pi\left|w_{m}\right|^{2} / a_{m}^{\prime} \leq \lambda(1-k)$.

Given this, we have $\pi\left|w_{1}\right|^{2} / a_{1}^{\prime}+\cdots+\pi\left|w_{n}\right|^{2} / a_{n} \leq \lambda(1-k)+k \leq \lambda$ and so $\phi(z)=w \in \lambda E\left(a_{1}^{\prime}, \ldots, a_{m}^{\prime}, a_{m+1}, \ldots, a_{n}\right)$ as required.

Statement (1) follows because on the region $\left\{\pi\left|z_{m+1}\right|^{2} / a_{m+1}+\cdots+\pi\left|z_{n}\right|^{2} / a_{n} \leq 1\right\}$ the Hamiltonian flow of $K$ preserves $r$ and hence $\pi\left|z_{m+1}\right|^{2} / a_{m+1}+\cdots+\pi\left|z_{n}\right|^{2} / a_{n}$.

For statement (2) note that if $\pi\left|z_{m+1}\right|^{2} / a_{m+1}+\cdots+\pi\left|z_{n}\right|^{2} / a_{n}=k$ then the partial derivatives of our Hamiltonian in the $z_{1}, \ldots, z_{m}$ directions are equal to the corresponding derivatives of $H_{1-k / \mu}\left(z_{1}, \ldots, z_{m}\right)$.

Now, $\left(z_{1}, \ldots, z_{m}\right) \in(1-k) E\left(a_{1}, \ldots, a_{m}\right) \subset(1-k / \mu) \mu E\left(a_{1}, \ldots, a_{m}\right)$. Thus as the flow of $H_{1-k / \mu}$ takes $(1-k / \mu) \mu E\left(a_{1}, \ldots, a_{m}\right) \rightarrow(1-k / \mu) \lambda E\left(a_{1}^{\prime}, \ldots, a_{m}^{\prime}\right)$ at time 1 we have $\left(w_{1}, \ldots w_{m}\right) \in(1-k / \mu) \lambda E\left(a_{1}^{\prime}, \ldots, a_{m}^{\prime}\right)$. In other words, $w$ satisfies $\pi\left|w_{1}\right|^{2} / a_{1}^{\prime}+\cdots+\pi\left|w_{m}\right|^{2} / a_{m}^{\prime} \leq(1-k / \mu) \lambda<\lambda(1-k)$ as claimed.

Remark 2.1 As mentioned in the introduction there is related work of Opshtein which we outline here, for convenience focusing on the case of embeddings into $\mathbb{C P}^{3}(c)$, complex projective space equipped with the Fubini-Study form scaled such that lines have symplectic area $c$. The symplectic manifold $\mathbb{C P}^{3}(c)$ is of special interest to us as the affine part is symplectomorphic to the 6-ball of capacity $c$. Opshtein observes the following. 
Theorem 2.2 (Opshtein [22]) Let $\Sigma \subset \mathbb{C P}^{3}(c)$ be a smooth holomorphic hypersurface of degree $k$. Then any symplectic embedding $E(a, b) \hookrightarrow \Sigma$ extends to a symplectic embedding $E(c / k, a, b) \hookrightarrow \mathbb{C P}^{3}(c)$.

For example, if $k=1$ then $\Sigma$ is a copy of $\mathbb{C P}^{2}$ which contains an embedded ball $B^{4}(c)$ of capacity $c$. Thus if $E(a, b) \hookrightarrow B(c)$ then we also find an embedding $E(c, a, b) \hookrightarrow$ $\mathbb{C P}^{3}(c)$. Under the same hypotheses our Proposition 1.1 also gives an embedding $E(c, a, b) \hookrightarrow B^{6}(c) \subset \mathbb{C P}^{3}(c)$. We expect the two embeddings are symplectically isotopic.

\subsection{Embedding obstructions}

To check that our constructions are sharp we rely only on the volume obstruction and on the Ekeland-Hofer capacities [6; 7]. The volume obstruction says the following.

Proposition 2.3 (Liouville's Theorem) If $E\left(a_{1}, \ldots, a_{n}\right) \hookrightarrow E\left(b_{1}, \ldots, b_{n}\right)$ then $a_{1} \cdots a_{n} \leq b_{1} \cdots b_{n}$.

The Ekeland-Hofer capacities give an infinite sequence of numbers $c_{k}\left(E\left(a_{1}, \ldots, a_{n}\right)\right)$ associated to an ellipsoid. In our situation we can take the definition to be as follows.

Definition $2.4 c_{k}\left(E\left(a_{1}, \ldots, a_{n}\right)\right)$ is the $k$-th number in the ordered sequence (with repetitions if necessary) of numbers in the set $\left\{k_{1} a_{1}, \ldots, k_{n} a_{n} \mid k_{i} \in \mathbb{N}\right\}$.

Theorem $2.5[6 ; 7] \quad$ If $E\left(a_{1}, \ldots, a_{n}\right) \hookrightarrow E\left(b_{1}, \ldots, b_{n}\right)$ then $c_{k}\left(E\left(a_{1}, \ldots, a_{n}\right)\right) \leq$ $c_{k}\left(E\left(b_{1}, \ldots, b_{n}\right)\right)$ for all $k$.

Note that $c_{k}\left(\lambda E\left(a_{1}, \ldots, a_{n}\right)\right)=\lambda c_{k}\left(E\left(a_{1}, \ldots, a_{n}\right)\right)$. Therefore if $E\left(a_{1}, \ldots, a_{n}\right) \hookrightarrow$ $E\left(b_{1}, \ldots, b_{n}\right)$ and $c_{k}\left(E\left(a_{1}, \ldots, a_{n}\right)\right)=c_{k}\left(E\left(b_{1}, \ldots, b_{n}\right)\right)$ for some $k$, we know that the embedding is optimal in the sense that there is no embedding $E\left(a_{1}, \ldots, a_{n}\right) \hookrightarrow$ $\mu E\left(b_{1}, \ldots, b_{n}\right)$ for any $\mu<1$.

\subsection{Some calculations in dimension 3}

Here we give some optimal embeddings for ellipsoids $E(1, a, b)$ when $a$ and $b$ are relatively small. We recall the definitions of the functions $f$ and $g$ from the introduction.

$$
\begin{aligned}
f(a, b) & :=\inf \left\{c \mid E(1, a, b) \hookrightarrow B^{6}(c)\right\}, \\
g(a) & :=\inf \left\{c \mid E(1, a) \hookrightarrow B^{4}(c)\right\} .
\end{aligned}
$$

The function $g$ is completely determined by McDuff and Schlenk [21]. In this paper we apply only a small amount of information about $g$ which is summarized in the following proposition. 
Proposition 2.6 (McDuff-Schlenk [21, Theorem 1.1.2]) If $b=4$ or $b>8 \frac{1}{36}$, then $g(b)=\sqrt{b}$.

We always assume without loss of generality that $1 \leq a \leq b$.

Lemma $2.7 f(g(b), b)=g(b)$.

Proof We observe that

$$
E(1, g(b), b) \cong E(g(b), 1, b) \hookrightarrow E(g(b), g(b), g(b))=B(g(b)),
$$

where the arrow follows from Proposition 1.1 and the definition of $g$. Therefore $f(g(b), b) \leq g(b)$.

Now, it follows from Proposition 2.6 that $g(b)=\sqrt{b}$ whenever $b \geq 9$. Thus the embedding is optimal in this case by Proposition 2.3.

If $b \leq 9$ then $g(b) \leq 3$. In the first case suppose that $1 \leq g(b) \leq 2$. Then

$$
c_{2}(E(1, g(b), b))=g(b)=c_{2}(B(g(b))) .
$$

In the second case, if $2 \leq g(b) \leq 3$ then

$$
c_{3}(E(1, g(b), b))=g(b)=c_{3}(B(g(b))) .
$$

Thus the embedding is also optimal in both of these cases by Theorem 2.5.

Lemma 2.8 Suppose that $a \leq 3$ and $g(b) \leq a$. Then $f(a, b)=a$.

Proof The embedding construction here is as in the previous proof of Lemma 2.7, and our hypothesis are such that the third Ekeland-Hofer capacity again implies that it is optimal.

Lemma 2.9 Suppose that $1 \leq a \leq b \leq 2$. Then $f(a, b)=b$.

Proof $E(1, a, b) \hookrightarrow B(b)$ simply by inclusion. But

$$
c_{3}(E(1, a, b))=b=c_{3}(B(b))
$$

for our range of $a, b$, so the inclusion is optimal.

Remark 2.10 It is true in any dimension that if $a_{n} \leq 2 a_{1}$ then $E\left(a_{1}, \ldots, a_{n}\right) \hookrightarrow B(c)$ if and only if $c \geq a_{n}$. This was established in the case of $n=2$ by Floer, Hofer and Wysocki in [8] as an application of symplectic homology. The theorem stated here is [23, Theorem 1], where Schlenk gives a simple proof by applying the $n$-th EkelandHofer capacity as in our proof of Lemma 2.9 above. We thank the referee for reminding us of this slightly strange history. 
Lemma 2.11 Suppose that $1 \leq a \leq 2 \leq b \leq 4$. Then $f(a, b)=2$.

Proof We have the embeddings

$$
E(1, a, b) \cong E(a, 1, b) \hookrightarrow E(a, 2,2) \hookrightarrow B(2)
$$

where the first arrow follows from Proposition 1.1 since $g(b)=2$ (see [21, Figure 1.1]) and the second arrow is the inclusion.

The third Ekeland-Hofer capacity

$$
c_{3}(E(1, a, b))=2=c_{3}(B(2))
$$

and so our construction is optimal.

We give one final computation which will rely on the following.

Lemma 2.12 $E(1,1,8) \hookrightarrow B(2)$.

Proof This is a particular case of Lemma 3.5 below.

Lemma 2.13 Suppose that $2 \leq b \leq 8$. Then $f(1, b)=2$.

Proof $f(1, b)$ is an increasing function of $b$. By Lemma 2.11, we know that $f(1,4)=2$ and Lemma 2.12 says that $f(1,8)=2$. Thus $f(1, b)$ is in fact constant on the interval $4 \leq b \leq 8$.

\section{Volume filling embeddings}

In this section we prove Theorem 3.8, a more precise version of Theorem 1.2. First, in Section 3.1 we recall two theorems of D McDuff on embeddings in dimension 4. The first reduces an ellipsoid embedding problem to one of embedding a disjoint union of balls into a ball, the second gives necessary conditions for embedding a disjoint union of balls. We close the subsection with two useful consequences. In Section 3.2 we use these theorems to derive some preliminary results on embedding ellipsoids in higher dimensions. We think these are quite interesting in themselves; the main result is Proposition 3.7 which will be applied in Section 4 to give our full packing result. Finally in Section 3.3 we prove Theorem 3.8. 


\subsection{Four dimensional embeddings}

Here we review some results of McDuff which allow one to translate a 4-dimensional ellipsoid embedding problem into one of a disjoint union of balls, and then give an algebraic solution for the ball embedding problem.

Proposition 3.1 (McDuff $[19 ; 16]$ ) Let $e, f, c, d$ be positive integers with $e \leq f$ and $c \leq d$. There exists a weight expansion $W(e, f)$ associated to any pair of integers such that a symplectic embedding of ellipsoids $E(e, f) \longrightarrow E(c, d)$ exists if and only if there exists a symplectic embedding of balls

$$
(\sqcup B(W(e, f))) \cup(\bigsqcup B(W(d-c, d))) \hookrightarrow B(d) .
$$

Let us explain the weight sequences $W(e, f)$, as they are defined for instance in [16].

$$
W(e, f)=\left(X_{0}^{\times l_{0}}, X_{1}^{\times l_{1}}, \ldots, X_{K}^{\times l_{K}}\right),
$$

where the multiplicities $l_{i}$ are the entries in the continued fraction expansion

$$
\frac{f}{e}=\left[l_{0} ; l_{1}, \ldots, l_{k}\right]=l_{0}+\frac{1}{l_{1}+\frac{1}{\ddots \cdot 1 / l_{k}}}
$$

and the entries $X_{i}$ are defined inductively as follows:

$$
X_{-1}=f, X_{0}=e, X_{i+1}=X_{i-1}-l_{i} X_{i} .
$$

We use the notation

$$
\sqcup B(W(e, f)):=\left(\bigsqcup_{i=1}^{l_{0}} B\left(X_{0}\right)\right) \cup\left(\bigsqcup_{i=1}^{l_{1}} B\left(X_{1}\right)\right) \cup \cdots \cup\left(\bigsqcup_{i=1}^{l_{K}} B\left(X_{K}\right)\right) .
$$

McDuff and Polterovich [20] equated the problem of embedding $M$ disjoint balls with understanding the symplectic cone of the $M$-fold blow-up $X_{M}$ of $\mathbb{C P}^{2}$. The structure of the cone was intensely studied by Biran [1] and later T-J Li and Liu [15] and B-H Li and T-J Li [13]. To explain their results, denote by $L, E_{1}, \ldots, E_{M} \in H_{2}\left(X_{m}, \mathbb{Z}\right)$ the homology classes of the line and the exceptional divisors, by $-K:=3 L-\sum_{i} E_{i}$ the anticanonical class, and by $l, e_{1}, \ldots, e_{M}$ their Poincaré duals. With respect to the basis $L,-E_{1}, \ldots,-E_{M}$ we represent homology classes by $(M+1)$-tuples $(d, \bar{m})$. We fix a symplectic form $\omega_{M}$ on $X_{M}$ obtained by symplectically blowing up $\left(\mathbb{C P}^{2}, \omega\right)$ with the standard Fubini-Study form $\omega$. Define $\mathcal{C}_{M}$ to be the set of all cohomology classes in $H^{2}\left(X_{M}\right)$ that can be represented by symplectic forms whose first Chern classes 
are Poincaré dual to $-K$. Next define the exceptional cone to be the set of homology classes $\mathcal{E}_{M} \subset H_{2}\left(X_{M}, \mathbb{Z}\right)$,

$\mathcal{E}_{M}:=\left\{E \mid E \cdot E=-1, E\right.$ is represented by an embedded $\omega_{M}$-symplectic sphere $\}$.

Since the classes $E$ in the exceptional cone have nontrivial Gromov invariants, the definition of the exceptional cone is independent of the choice of $\omega_{M} . \mathrm{B}-\mathrm{H} \mathrm{Li}$ and T-J Li [13] showed that

$$
\mathcal{C}_{M}=\left\{\alpha \in H^{2}\left(X_{M}\right) \mid \alpha^{2}>0 \text { and } \alpha(E)>0 \text { for all } E \in \mathcal{E}_{M} t\right\} .
$$

Given these definitions, the work of McDuff and Polterovich, Biran, T-J Li and Liu and $\mathrm{B}-\mathrm{H} \mathrm{Li}$ and T-J Li, gives the following criteria for embedding disjoint unions of balls.

Proposition $3.2 \quad[20 ; 1 ; 13 ; 14 ; 15]$ A symplectic ball embedding

$$
\bigsqcup_{i=1, \ldots, M} B^{4}\left(w_{i}\right) \hookrightarrow B^{4}(\mu)
$$

exists if, and only if, $\mu l-\sum_{i=1}^{M} w_{i} e_{i} \in \mathcal{C}_{M}$. This is equivalent to the following two conditions.

(1) $\mu^{2} \geq \sum_{i=1}^{M} w_{i}^{2}$.

(2) For any $\left(d^{\prime}, \bar{m}\right) \in \mathcal{E}_{M}$, we have

$$
d^{\prime} \mu \geq \sum_{i=1}^{M} m_{i} w_{i}
$$

We close this subsection with the following two applications of these results.

Proposition 3.3 For any $k, x \in \mathbb{N}$, the following embedding holds:

$$
E\left(1, k^{2 x+1}\right) \hookrightarrow E\left(k^{x}, k^{x+1}\right) .
$$

Proof We apply Proposition 3.1 with $e=1, f=k^{2 x+1}, c=k^{x}, d=k^{x+1}$. Then the equivalent embedding (2) (see the notation (5)) becomes

$$
\sqcup B\left(W\left(1, k^{2 x+1}\right)\right) \cup \sqcup B\left(W\left(k^{x+1}-k^{x}, k^{x+1}\right)\right) \hookrightarrow B\left(k^{x+1}\right) .
$$

The continuous fraction expansions are $1 / k^{2 x+1}=\left[0 ; k^{2 x+1}\right]$ and $\left(k^{x+1}-k^{x}\right) / k^{x+1}=$ $[0 ; 1, k-1]$, so the vectors described in (4) express this last embedding as

$$
\left(\bigsqcup_{i=1}^{k^{2 x+1}} B_{i}(1)\right) \cup B\left(k^{x+1}-k^{x}\right) \cup\left(\bigsqcup_{j=1}^{k-1} B\left(k^{x}\right)\right) \hookrightarrow B\left(k^{x+1}\right) .
$$

This embedding can be seen from a toric viewpoint. Indeed, there exists a toric decomposition of the ball of capacity $k^{x+1}$ containing an open ball of capacity $k^{x+1}-k^{x}$ 
and the preimages of $2 k-1$ polytopes of capacity $k^{x}$ as shown in Figure 2. For the fact that these open toric manifolds admit embeddings of an open ball of the stated capacity, see Traynor [25, Proposition 5.2]. But a ball of capacity $k^{x}$ can be filled with $k^{2 x}$ balls of capacity 1; see [25, Construction 3.2] for an explicit construction. If we decompose $k$ of our $2 k-1$ such balls in this way then we get the embedding as required.

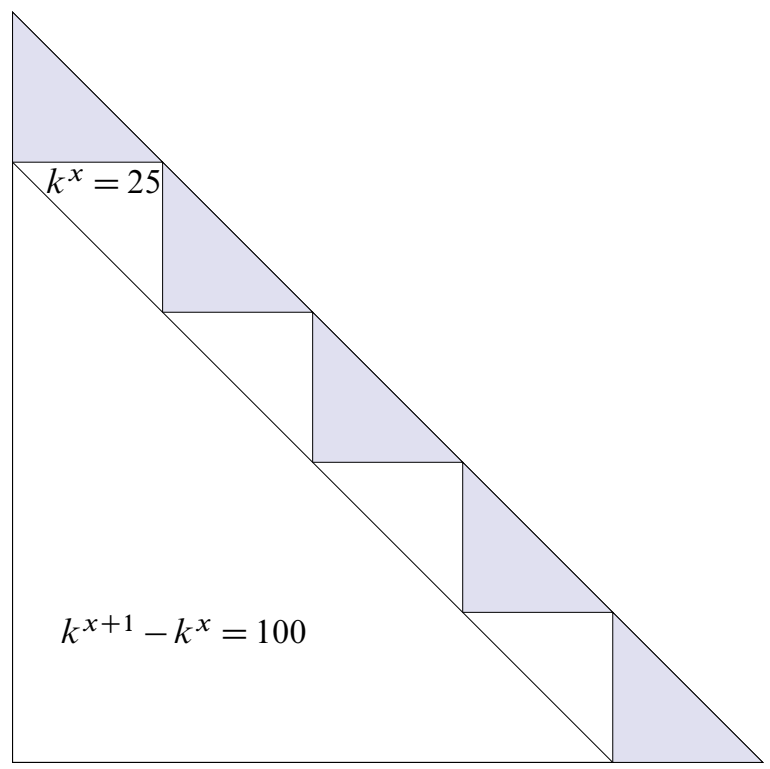

Figure 2: A toric decomposition of the ball when $k=5, x=2$. The same toric packing strategy applies to any $k, x$ natural.

This concludes the proof of Proposition 3.3.

Lemma 3.4 If $\sqrt{2 / 3} \leq \lambda \leq 1$ and $b \geq 9$, then $E(1, b) \hookrightarrow E\left(\lambda \sqrt{b}, \lambda^{-1} \sqrt{b}\right)$.

Proof The proof largely follows the method used by McDuff and Schlenk in [21, Corollary 1.2.4] where they establish the case $\lambda=1$. It is sufficient to consider the case when both $\lambda=u / v$ and $\sqrt{b}=p / q$ rational, with $p \geq 3 q, \sqrt{2 / 3} \leq u / v \leq 1$. Then we need to prove that

$$
E\left(1, \frac{p^{2}}{q^{2}}\right) \hookrightarrow E\left(\frac{u p}{v q}, \frac{v p}{u q}\right)
$$

and the latter is equivalent to showing that there exists an embedding

$$
E\left(u v p^{2}, u v q^{2}\right) \hookrightarrow E\left(u^{2} p q, v^{2} p q\right) .
$$


Using now Proposition 3.1 we see that this is equivalent to the existence of the following ball embedding:

$$
\left(\bigsqcup B\left(W\left(u v q^{2}, u v p^{2}\right)\right)\right) \cup\left(\bigsqcup B\left(W\left(\left(v^{2}-u^{2}\right) p q, v^{2} p q\right)\right)\right) \hookrightarrow B\left(v^{2} p q\right) .
$$

We will use the criteria from Proposition 3.2 to show that the embedding (12) does indeed exist.

The first condition is clearly satisfied because our embeddings are volume preserving. To verify the second, note that since $\left(d^{\prime}, \bar{m}\right) \in \mathcal{E}_{M}$ we have $3 d^{\prime}-1=\sum_{i=1}^{M} m_{i}$.

Let

$$
\bigsqcup B\left(W\left(u v q^{2}, u v p^{2}\right)\right)=\bigsqcup_{i=1}^{M_{1}} B\left(w_{i}\right),
$$

and

$$
\sqcup B\left(W\left(\left(v^{2}-u^{2}\right) p q, v^{2} p q\right)\right)=\bigsqcup_{i=M_{1}+1}^{M} B\left(w_{i}^{\prime}\right) .
$$

We have

$$
\sum_{i=1}^{M_{1}} m_{i} w_{i}+\sum_{i=M_{1}+1}^{M} m_{i} w_{i}^{\prime} \leq \sum_{i=1}^{M_{1}} u v q^{2} m_{i}+\sum_{i=M_{1}+1}^{M}\left(\left(v^{2}-u^{2}\right) p q\right) m_{i}
$$

since each $w_{i} \leq u v q^{2}$ and each $w_{i}^{\prime} \leq\left(v^{2}-u^{2}\right) p q$. Therefore

$$
\begin{aligned}
\sum_{i=1}^{M_{1}} m_{i} w_{i}+\sum_{i=M_{1}+1}^{M} m_{i} w_{i}^{\prime} & \left.\leq \max \left\{u v q^{2},\left(v^{2}-u^{2}\right) p q\right)\right\} \sum_{i=1}^{M} m_{i} \\
& \left.=\max \left\{u v q^{2},\left(v^{2}-u^{2}\right) p q\right)\right\}\left(3 d^{\prime}-1\right) .
\end{aligned}
$$

Thus, to show the inequality (6), it is sufficient to verify that both of the following inequalities hold:

$$
\begin{aligned}
u v q^{2}\left(3 d^{\prime}-1\right) & \leq v^{2} p q d^{\prime}, \\
\left(v^{2}-u^{2}\right) p q\left(3 d^{\prime}-1\right) & \leq v^{2} p q d^{\prime} .
\end{aligned}
$$

The first inequality is guaranteed if $3 u v q^{2} \leq v^{2} p q$, or $u / v \leq(1 / 3)(p / q)$. This is certainly true as $\lambda \leq 1$ and $\sqrt{b} \geq 3$.

The second is guaranteed if $3\left(v^{2}-u^{2}\right) p q \leq v^{2} p q$, or, $u^{2} / v^{2} \geq 2 / 3$. This is true as well since we assumed $\lambda^{2} \geq 2 / 3$.

\subsection{Applications to higher dimensions}

We start this section by applying Proposition 3.3 to obtain embeddings in higher dimensions.

For brevity, we will use the notation $E\left(a_{1}, a_{2}, \ldots, a_{i}^{\times m}, a_{i+m}, \ldots a_{n}\right)$ if an entry $a_{i}$ is repeated $m$ times. 
Lemma 3.5 For any $k, n \in \mathbb{N}$,

$$
E\left(1^{\times(n-1)}, k^{n}\right) \hookrightarrow B(k) .
$$

Proof We fix $k$ and proceed by induction on $n$. First, (17) is obviously true when $n=1$, while the case $n=2$, first proved in [19], can be easily seen as part of McDuff and Schlenk's results on the values of $g(b)$ from Proposition 2.6.

Let $n \geq 3$. If $n$ is odd, say $n=2 m+1$, we have

$$
E\left(1^{\times(n-1)}, k^{n}\right) \hookrightarrow E\left(1^{\times(n-2)}, k^{m}, k^{m+1}\right)=E\left(1^{\times(m-1)}, k^{m}, 1^{\times m}, k^{m+1}\right),
$$

where the first embedding is from Proposition 3.3. By the induction hypothesis and Proposition 1.1, the final ellipsoid embeds into

$$
E\left(k^{\times m}, k^{\times(m+1)}\right)=E\left(k^{\times n}\right) .
$$

On the other hand, if $n$ is even, say $n=2 m$, then

$$
E\left(1^{\times(n-1)}, k^{n}\right)=E\left(1^{\times m}, 1^{\times(m-1)},\left(k^{2}\right)^{m}\right),
$$

which by the induction hypothesis (with $k^{2}, m$ instead of $k, n$ ) and Proposition 1.1 again embeds into

$$
E\left(1^{\times m},\left(k^{2}\right)^{\times m}\right) .
$$

But by using Proposition 1.1 repeatedly and the fact that $E\left(1, k^{2}\right) \hookrightarrow E(k, k)$ (see again Proposition 2.6) we can split every instance of $k^{2}$ into two copies of $k$, which ends the proof.

Next, Lemma 3.4 will be used in order to prove a similar result to Lemma 3.5, but replacing $k^{n}$ with a real number.

Definition 3.6 Define the sequence $M_{i}, i \geq 2$ inductively as follows: $M_{2}=8 \frac{1}{36}$, $M_{n}=\max \left(M_{n-1}^{2}, \beta_{n}\right)$, where

$$
\beta_{n}=\left(\frac{\sqrt[2 n-2]{3}}{\sqrt[2 n-2]{3}-\sqrt[2 n-2]{2}}\right)^{2 n(n-1) /(n-2)}
$$

We are now in a position to state the main result of this subsection.

Proposition 3.7 If $b$ is any real number with $b \geq M_{n}$, then $E\left(1^{\times(n-1)}, b\right) \hookrightarrow B\left(b^{1 / n}\right)$. 
Proof We will proceed by induction with respect to the dimension $n$. The result holds when $n=2$ by [21]; see Proposition 2.6. Assume that the result holds for ellipsoids of complex dimension less or equal than $n-1$.

We set

$$
\lambda=\left(\frac{\left\lfloor b^{(n-2) /(2 n(n-1))}\right\rfloor}{b^{(n-2) /(2 n(n-1))}}\right)^{n-1} .
$$

Then since $b \geq \beta_{n}$ we observe that

$$
\sqrt{\frac{2}{3}} \leq\left(\frac{b^{(n-2) /(2 n(n-1))}-1}{b^{(n-2) /(2 n(n-1))}}\right)^{n-1} \leq \lambda \leq 1 .
$$

By Lemma 3.4 and Proposition 1.1 we have the embedding

$$
E\left(1^{\times(n-1)}, b\right) \hookrightarrow E\left(1^{\times(n-2)}, \lambda^{-1} \sqrt{b}, \lambda \sqrt{b}\right) .
$$

Meanwhile, since $\lambda^{-1} \sqrt{b} \geq M_{n-1}$, by the induction hypothesis we have the embedding

$$
\begin{aligned}
E\left(1^{\times(n-2)}, \lambda^{-1} \sqrt{b}, \lambda \sqrt{b}\right) \hookrightarrow E\left(\left(\left(\lambda^{-1} \sqrt{b}\right)^{1 /(n-1)}\right)^{\times(n-1)}, \lambda \sqrt{b}\right) \\
=\left(\lambda^{-1} \sqrt{b}\right)^{1 /(n-1)} E\left(1^{\times(n-1)}, z\right),
\end{aligned}
$$

where $z=\frac{\lambda \sqrt{b}}{\left(\lambda^{-1} \sqrt{b}\right)^{1 /(n-1)}}=\left(\lambda^{n /(n-1)}\right) \cdot\left(b^{(n-2) /(2 n-2)}\right)=\left\lfloor b^{(n-2) /(2 n(n-1))}\right\rfloor^{n}$.

Thus our result follows from the corresponding result for integers, Lemma 3.5.

\subsection{Proof of Theorem 1.2}

Here we show the following.

Theorem 3.8 Suppose that

or

$$
\begin{gathered}
b>\left(M_{3}\right)^{4} a^{2} \\
a \geq 8 \frac{1}{36} \quad \text { and } \quad b>\left(M_{3}\right)^{2} .
\end{gathered}
$$

Then $E(1, a, b) \hookrightarrow B\left((a b)^{1 / 3}\right)$.

Remark 3.9 As $1 \leq a \leq b$, the hypotheses of Theorem 3.8 are automatically satisfied if

$$
a^{2}+b^{2} \geq\left(8 \frac{1}{36}\right)^{2}\left(1+\left(8 \frac{1}{36}\right)^{2}\left(\frac{\sqrt[4]{3}}{\sqrt[4]{3}-\sqrt[4]{2}}\right)^{96}\right)>1.41 \times 10^{101}
$$


Proof First suppose that $b>\left(M_{3}\right)^{4} a^{2}$. We note that this automatically implies that $b>8 \frac{1}{36}$ and $\sqrt{b} / a>8 \frac{1}{36}$. Then we have

$$
\begin{aligned}
E(1, a, b) \hookrightarrow & E(a, \sqrt{b}, \sqrt{b}) \\
& =a E(1, \sqrt{b} / a, \sqrt{b} / a) \hookrightarrow a E\left(b^{1 / 4} / a^{1 / 2}, b^{1 / 4} / a^{1 / 2}, \sqrt{b} / a\right) \\
& =a^{1 / 2} b^{1 / 4} E\left(1,1, b^{1 / 4} / a^{1 / 2}\right) \hookrightarrow a^{1 / 2} b^{1 / 4} B\left(b^{1 / 12} / a^{1 / 6}\right) \\
& =B\left((a b)^{1 / 3}\right) .
\end{aligned}
$$

The first embedding is possible since $b>8 \frac{1}{36}$, the second since $\sqrt{b} / a>8 \frac{1}{36}$, and the third by Proposition 3.7 since $b^{1 / 4} / a^{1 / 2}>M_{3}$.

Next we assume that $a \geq 8 \frac{1}{36}$ and $b>\left(M_{3}\right)^{2}$. Then we have embeddings

$$
E(1, a, b) \hookrightarrow E(\sqrt{a}, \sqrt{a}, b)=\sqrt{a} E(1,1, b / \sqrt{a}) \hookrightarrow \sqrt{a} B\left(b^{1 / 3} / a^{1 / 6}\right)=B\left((a b)^{1 / 3}\right) .
$$

Now the first embedding relies on $a \geq 8 \frac{1}{36}$ and the second exists by Proposition 3.7 again, since $b / \sqrt{a}>\sqrt{b}>M_{3}$.

\section{Packing stability in $\mathbb{C P}^{n}$}

In this section we prove Theorem 1.3. It relies on the following generalization to higher dimensions of a 4-dimensional polytope decomposition introduced by McDuff in [19].

Lemma 4.1 For any $k \in \mathbb{N}$,

$$
\bigsqcup_{k} B(1) \hookrightarrow E\left(1^{\times(n-1)}, k\right) .
$$

That is, for any $\rho<1$ the disjoint union of $k$ balls of capacity $\rho$ can be symplectically embedded in the ellipsoid $E\left(1^{\times(n-1)}, k\right)$.

Remark 4.2 Lemma 2.6 of [19] proves a stronger result in dimension 4, namely that there exists a symplectic embedding $\bigsqcup_{k} \stackrel{\circ}{B}^{4}(1) \rightarrow \stackrel{\circ}{E}(1, k)$ from the interiors of the balls to the interior of the ellipsoid. As far as we know, it is unknown if such an embedding exists in higher dimension. Nevertheless, we give a proof of Lemma 4.1 following this lemma.

There is an alternative approach following Traynor [25, Chapters 5 and 6]; see also [23, Section 9.4]. The idea is to approximate both the ellipsoid and the balls by Lagrangian products. Once this is done correctly it is easy to see that at least an arbitrarily large compact subset of the $k$ open balls can be symplectically embedded in the ellipsoid. 
Proof of Lemma 4.1 There is a natural action of the torus $T^{n}$ on the open ellipsoid $\stackrel{\circ}{E}\left(1^{\times(n-1)}, k\right) \subset \mathbb{C}^{n}$ given by rotation in each of the $n$ complex coordinates. Let $\left\{\boldsymbol{e}_{j}\right\}$ denote the standard basis in $\mathbb{R}^{n}$. Then the moment polytope $\Delta$ of the corresponding moment map is the convex hull of the set $\left\{0, \boldsymbol{e}_{1}, \boldsymbol{e}_{2}, \ldots, \boldsymbol{e}_{n-1}, k \boldsymbol{e}_{n}\right\}$ minus the diagonal; that is, the $n$-dimensional polytope of vectors $p=\left(x_{1}, \ldots, x_{n}\right)$ that satisfy

$$
x_{j} \geq 0 \quad \text { and } \quad x_{1}+\cdots+x_{n-1}+x_{n} / k<1 .
$$

Inside $\Delta$ we can find $k$ subpolytopes $\Delta_{j}$ defined by taking the interior of the convex hull of $\left\{\boldsymbol{e}_{1}, \ldots, \boldsymbol{e}_{n-1},(j-1) k \boldsymbol{e}_{n}, j k \boldsymbol{e}_{n}\right\}$ for $j=1 \ldots k$; see Figure 3. The affine map $\Theta: \mathbb{R}^{n} \longrightarrow \mathbb{R}^{n}$ that fixes the first $n-1$ coordinates and takes the $n$-th coordinate $x_{n}$ to $x_{n}-\left(k-\left(x_{1}+\cdots+x_{n-1}\right)\right)$ has integer coefficients and takes each $\Delta_{j}$ onto the previous polytope $\Delta_{j-1}$ so that each $\Delta_{j}$ maps onto $\Delta_{1}$ by the integral affine map $\Theta^{j-1}$.

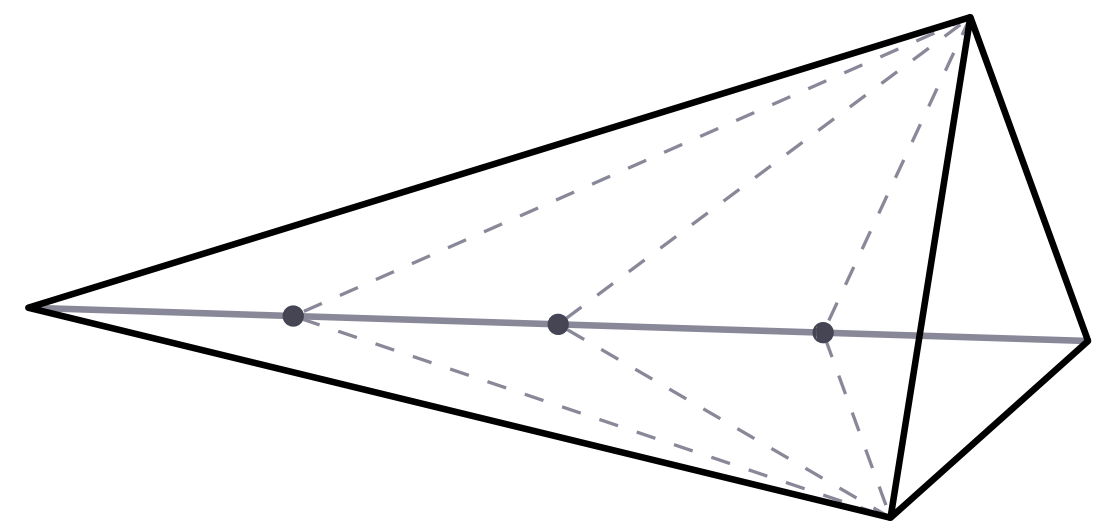

Figure 3: Division of the polytope $\Delta$

Now, one can see that $\Delta_{1}$ is the moment polytope of the open subset

$$
U=\left\{\left.\left(z_{1}, \ldots, z_{n}\right) \in \mathbb{C}^{n}\left|z_{i} \neq 0, \pi\right| z_{1}\right|^{2}+\cdots+\pi\left|z_{n}\right|^{2}<1\right\} \subset B(1) \subset \mathbb{C P}^{n} .
$$

This contains an embedded symplectic open ball of capacity $\rho$ for any $\rho<1$, for this, one can apply a product map such as $\Psi^{\rho}$ defined in [25, page 420].

From the above, all other $\Delta_{j}$ must also admit an embedding of a ball of the same capacity. Thus, we have found an embedding

$$
\bigsqcup_{k} B(\rho) \rightarrow E\left(1^{\times(n-1)}, k\right)
$$

for any $\rho<1$, as required. 
Remark 4.3 The result above enables us to find embeddings of disjoint balls into a space whenever ellipsoid embeddings of the specified type are available. Theorem 1.1 in [19] also gives a converse, that an ellipsoid embedding exists whenever the corresponding ball embedding does. This result uses an orbifold blow up as in Godinho [9] (see also McDuff [18, Section 2.2]) then the theory of holomorphic curves and the rational blow down constructions due to Symington [24]. No such technology is yet available in higher dimension.

Proof of Theorem 1.3 Rescaling, it is required to show that for all natural numbers $k \geq M_{n}$ there exists an embedding

$$
\bigsqcup_{k} B(1) \hookrightarrow B^{2 n}\left(k^{1 / n}\right) .
$$

By Proposition 3.7 there exists an embedding

$$
E\left(1^{\times(n-1)}, k\right) \hookrightarrow B\left(k^{1 / n}\right),
$$

so it suffices to find an embedding

$$
\bigsqcup_{k} B(1) \hookrightarrow E\left(1^{\times(n-1)}, k\right) .
$$

But this is Lemma 4.1.

Remark 4.4 (Improving the bounds) Our stability bound $M_{n}$ in Theorem 1.3 and Theorem 1.4 seem far from optimal. Improving these bounds will be the topic of a future work [4]. This will follow from improving the bound $M_{n}$ in Proposition 3.7. Now, according to [16], one 4-dimensional ellipsoid embeds into another if and only if there are no obstructions coming from the Embedded Contact Homology capacities defined by M Hutchings in [11]. Therefore we have a route to proving Proposition 3.3 for real rather than just integer $k$ by studying the behavior of the ECH capacities. Once this is done, Proposition 3.7 follows exactly as Lemma 3.5.

\section{References}

[1] P Biran, Symplectic packing in dimension 4, Geom. Funct. Anal. 7 (1997) 420-437 MR1466333

[2] P Biran, A stability property of symplectic packing, Invent. Math. 136 (1999) 123-155 MR1681101

[3] P Biran, From symplectic packing to algebraic geometry and back, from: "European Congress of Mathematics, Vol. II (Barcelona, 2000)", (C Casacuberta, R M Miró-Roig, J Verdera, S Xambó-Descamps, editors), Progr. Math. 202, Birkhäuser, Basel (2001) 507-524 MR1909952 
[4] O Buse, R Hind, Optimal higher dimensional ellipsoids embeddings, in preparation

[5] K Cieliebak, H Hofer, J Latschev, F Schlenk, Quantitative symplectic geometry arXiv:math.SG/0506191

[6] I Ekeland, H Hofer, Symplectic topology and Hamiltonian dynamics, Math. Z. 200 (1989) 355-378 MR978597

[7] I Ekeland, H Hofer, Symplectic topology and Hamiltonian dynamics II, Math. Z. 203 (1990) 553-567 MR1044064

[8] A Floer, H Hofer, K Wysocki, Applications of symplectic homology I, Math. Z. 217 (1994) 577-606 MR1306027

[9] L Godinho, Blowing up symplectic orbifolds, Ann. Global Anal. Geom. 20 (2001) 117-162 MR1857175

[10] M Gromov, Pseudoholomorphic curves in symplectic manifolds, Invent. Math. 82 (1985) 307-347 MR809718

[11] M Hutchings, Quantitative embedded contact homology, to appear in J. Differential Geom. arXiv: 1005.2260

[12] F Lalonde, D McDuff, The classification of ruled symplectic 4-manifolds, Math. Res. Lett. 3 (1996) 769-778 MR1426534

[13] B-H Li, T-J Li, Symplectic genus, minimal genus and diffeomorphisms, Asian J. Math. 6 (2002) 123-144 MR1902650

[14] B-H Li, T-J Li, On the diffeomorphism groups of rational and ruled 4-manifolds, J. Math. Kyoto Univ. 46 (2006) 583-593 MR2311360

[15] T-J Li, A-K Liu, Uniqueness of symplectic canonical class, surface cone and symplectic cone of 4-manifolds with $B^{+}=1$, J. Differential Geom. 58 (2001) 331-370 MR1913946

[16] D McDuff, The Hofer conjecture on embedding symplectic ellipsoids, to appear in J. Differential Geom. arXiv: 1008.1885v3

[17] D McDuff, From symplectic deformation to isotopy, from: "Topics in symplectic 4manifolds (Irvine, CA, 1996)", (R J Stern, editor), First Int. Press Lect. Ser. I, Int. Press, Cambridge, MA (1998) 85-99 MR1635697

[18] D McDuff, Some 6-dimensional Hamiltonian $S^{1}$-manifolds, J. Topol. 2 (2009) 589623 MR2546587

[19] D McDuff, Symplectic embeddings of 4-dimensional ellipsoids, J. Topol. 2 (2009) 1-22 MR2499436

[20] D McDuff, L Polterovich, Symplectic packings and algebraic geometry, Invent. Math. 115 (1994) 405-434 MR1262938 With an appendix by Y Karshon

[21] D McDuff, F Schlenk, The embedding capacity of 4-dimensional symplectic ellipsoids arXiv:0912.0532v2 
[22] E Opshtein, Maximal symplectic packings in $\mathbb{P}^{2}$, Compos. Math. 143 (2007) 15581575 MR2371382

[23] F Schlenk, Embedding problems in symplectic geometry, de Gruyter Exp. in Math. 40, de Gruyter, Berlin (2005) MR2147307

[24] M Symington, Symplectic rational blowdowns, J. Differential Geom. 50 (1998) 505518 MR1690738

[25] L Traynor, Symplectic packing constructions, J. Differential Geom. 42 (1995) 411-429 MR1366550

Department of Mathematics, Indiana University Purdue University Indianapolis

Indianapolis IN 46202, USA

Department of Mathematics, University of Notre Dame

Notre Dame IN 46556, USA

buse@math.iupui.edu, hind.1@nd.edu

Proposed: Leonid Polterovich

Received: 18 April 2011

Seconded: Yasha Eliashberg, Ronald Fintushel

Revised: 16 August 2011 